\title{
The Physics of the Ideal Continuum
}

\author{
Vedran Furtula ${ }^{1}$ \\ ${ }^{1}$ Individual Researcher, MSc
}

\begin{abstract}
In this paper from the domain of theoretical physics, there have been stated and briefly described the basic principles and laws that apply to the new scientific discipline of physics, which we call the Physics of the Ideal Continuum. In addition to the definition of the ideal continuum, it also defines and describes other phenomena such as the origin of matter, the origin of space-time, the origin and role of black holes and other phenomena in the universe. Special attention has been focused on the energy balance in the universe, as well as on the universal physical constant and its role in the development of the mathematical and physical models of the universe. Through the presentation of the differences between the physics of the ideal continuum, the quantum physics and classical physics, an additional stride has been made in understanding the most important laws and their applicability in these scientific disciplines, as well as their inter-connectedness. Other terms that have been given a significant role in this paper include equilibrium and gravitationalsheds (gravitational divisions).
\end{abstract}

Keywords:- The Physics of the Ideal Continuum, ideal continuum, energy balance in the universe, universal physical constant, equilibrium, gravitationalsheds.

\section{INTRODUCTION}

Today, when we fully understand classical physics of matter and easily use the established laws of motion of material bodies in space-time, and when we have seriously stepped into quantum physics of antimatter bodies or the energy of material particles at the atomic and subatomic level, or a new branch of physics, which has given classical physics a completely new dimension, we can rightfully say that we are close to major breakthroughs in the development of these fundamental scientific disciplines of a unique science - physics. Many scientists of the modern era in which we live, have made an immeasurable contribution to understanding and uniting these two scientific disciplines with the universal laws of physics, but still without success in the end.

This paper in the field of theoretical physics deals with a new scientific discipline that we will call the physics of the ideal continuum. The goal of this new scientific discipline is not to reconcile the opinions of prominent physicists of classical and quantum physics, nor to deny or refute them in any way, but, on the contrary, by analysing basic physical quantities such as time, space, matter, energy, light, gravity and many others, including the physical constants established so far, its goal is to attempt to define and describe their properties, behaviour, and laws in something what we call the (absolutely) ideal continuum.

\section{THE IDEAL CONTINUUM}

To begin with and to get a better understanding, the ideal continuum will be called absolutely empty space (like the Dirac vacuum) or the space absolutely filled with an absolutely ideal homogeneous isotropic antimatter or matter (if such matter exists). The ideal continuum has all the properties of the ideal medium, i.e. the space no any properties of which affect in any way any process that takes place in it. In other words, the ideal continuum is the initial, zero state of space, free of any (physical) properties. Similarly, the ideal continuum can equally has of micro (infinitesimal), measurable and macro (infinite) dimensions and scale.

The physical phenomena that can be compared or equated with the ideal continuum are the so-called black holes, quasars, dark energy and antimatter (which the most part of the universe is filled with), the initial (zero) state of the universe (regardless of the momentum: before expansion or before the so-called Big Bang, after the complete disintegration of the universe, or after the total inflation and absolute space-time compression), as well as the empty atomic and subatomic space and fields within material and antimatter particles.

In order to draw adequate theoretical conclusions, it is also necessary to define other scientific starting points and conditions, which will not only simplify the task, but also make it more understandable and more accessible to the wider scientific public, as well as enable its simpler confirmation through direct astronomical observations and other scientific experiments. First of all, it is necessary to describe the way in which some of the basic physical occurrences and phenomena in the universe happen, as well as the way in which they cause other physical phenomena and processes thereof in the cause-and-effect relationships. In the following chapters, one of the basic hypotheses about the origin of the universe, or the socalled Big Bang theory [1] will be put forward and described.

\section{THE UNIVERSE AND MULTI-VERSE}

Under the term universe, we will encompass the entire visible and invisible universe or multi-verse, regardless of whether it exists/they exist simultaneously or whether their existences are cause-and-effect related in any way. Therefore, here we consider the universe as a general term for the entire and absolute space in the entire and absolute time. 


\section{INTERFERENCE AND THE SPEED OF LIGHT}

In this paper, we will exclusively analyse the basic property of a quantum, as an elementary unit of energy, i.e. the photon interference. Based on the already proven physical properties of the photon and its ability to be in two or more places at the same time (both a particle and a wave simultaneously), we will at this very moment draw one of the main conclusions, which is, the speed of light in a vacuum is not the maximum possible (limit) speed achieved, but antimatter particles can achieve even higher speed than the speed of light (1).

$$
\left|v_{\max }\right| \gg c
$$

\section{OBSERVER'S REFERENCE SYSTEM}

In accordance with the relativistic laws of physics, what conclusions we will draw about a physical process depends on which system we declare as the reference. There is a big difference if the observer is in the centre of a black hole, its immediate surroundings or at the very edge of its zone of influence - gravitationalsheds (Figure 1).

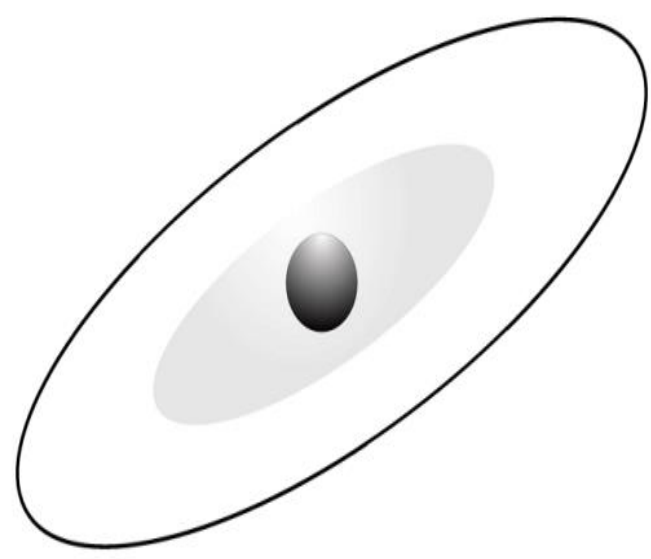

Fig 1:- Gravitationalsheds (of the system, galaxy, ideal continuum or the universe)

There is also a difference if the observer is in a galaxy, a cluster of galaxies or the universe, which is in the phase of collapse and inflation, while the rest of the multiverse is in the phase of expansion and disintegration. One example to describe the said phenomenon is several balloons in each other, the interior one of which, whose centre has been determined as the reference system, collapses, while the other one, the outer balloon, continues expanding rapidly, and doing it on alternately. If we went a step further and reversed the processes realising that the interior balloon was expanding and the outer balloon shrinking, we would then have a bigger problem understanding and describing the physical phenomena in such complex systems (Figure 2).

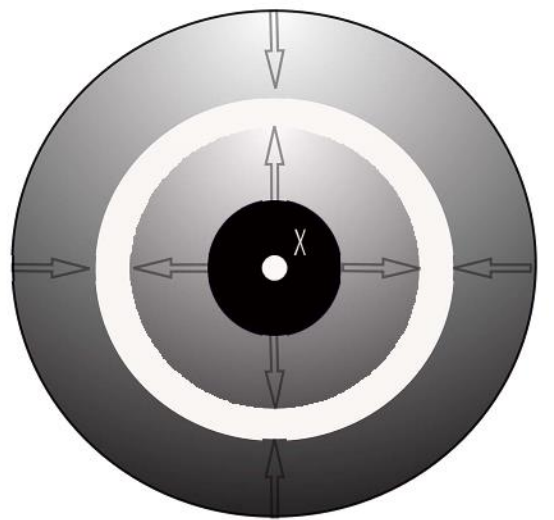

Fig 2:- Reference System

The position of our reference system, from which we observe and try to describe the entire visible and invisible universe (Figure 3), has caused the need for most of the mentioned physical constants (Cosmological, Hubble's, etc.), with which we basically try to correct the equations or calibrate the developed mathematical and physical models of the universe.

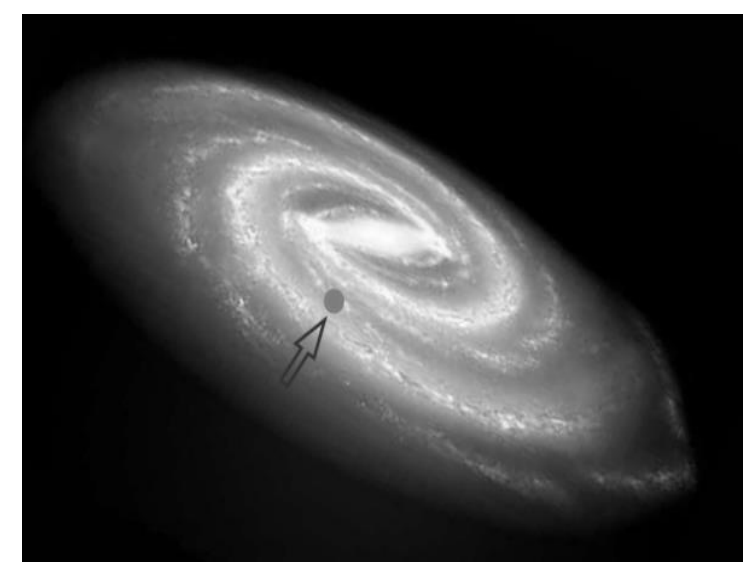

Fig 3:- The position of our reference system (The Solar System in the Milky Way galaxy)

\section{PHYSICAL CONSTANTS}

Planck's constant, Planck's mass, Planck's length, Dirac's constant, Gravitational constant, Cosmological constant, Hubble's constant, etc. are the ones that play the most substantial role in physics of the all physical constants in general, as well as in the physics of the ideal continuum. The largest number of physical constants is directly correlated with the space-time distortion, especially in the reference system in relation to which we are trying to describe and understand all visible and invisible physical phenomena in the entire universe. Physical constants, as well as energy and matter, reach their finite values, values of zero or infinite values, if they are found or analysed in the immediate vicinity, i.e. at a significant (outside the direct influence) distance from the ideal continuum. In addition, in the physics of the ideal continuum, the largest number of physical constants should be absolutely ignored, they annihilate themselves or one another mutually. Based on the presented views on physical constants, as well as their arithmetic errors, we will establish the second 
conclusion of this paper, which is, the physics of the ideal continuum is actually the physics of a medium and information and not matter or antimatter particles (quantum, energy), and that or such a system (out of reference system), physical constants have no special or almost no role (2).

$$
\mid \text { const. } \mid \cong 0
$$

\section{BLACK HOLES AND QUASARS}

We will describe black holes, among other things, as physical phenomena in the universe that occur when the appropriate (physical) conditions are met. They represent essential disintegrators of matter into all visible and invisible forms of energy, as well as its occurring derivatives/products, of which we will list only those of interest to this paper, i.e. radiation, (gravitational and other) waves, heat and light. Black holes are the ideal medium and conductor (the ideal continuum), and as such, a place where antimatter particles reach/achieve higher velocities than the speed of light. Through the widespread dark energy and antimatter in the universe (continuum of medium), based on interference, the simultaneous appearance of information (regardless of the relativistic concept of time, as well as its division into past, present and future) in all black holes and all parts of the universe is possible. As the basic source of radiation and waves in the universe, due to the space-time compression, black holes also represent points at which time loses the sense of existence (as it does in all types of the ideal continuum), i.e. it equates with zero (3). A special forms of super-massive black holes, for which we apply the same laws that will be established in this paper, are quasars (visible disintegrators).

$$
|t|=0
$$

\section{TIME}

We have acquired the general notion of time through the understanding of gravity and classical (Newtonian) physics and mechanics of matter bodies. With the further development of physics, i.e. the established (Einstein's) theories of relativity, time has become a relative category, i.e. physical quantity that depends on the location of the observer and the position of the (reference) system in which it is located. Thus, the calculation of time or any physical process in other neighbouring systems, in relation to the reference, using the fundamental principle of the speed of light as absolute and maximum, as well as physical constants, is possible to carry out with a high degree of accuracy.

The physics of the ideal continuum is the physics in which time is equal to zero (3). It is the physics in which other physical quantities, including the basic ones, too, velocity or speed (4), acceleration, energy, etc., reach their maximum, infinite values or, in the case of mass, their minimum values (5). This is the physics in which the size of space plays absolutely no role, for it is only a medium filled with the ideal continuum, through which, and in which, a piece of information, as the final product of energy disintegration, encounters no resistance.

$$
\begin{aligned}
& |v|=\infty \\
& |m|=0
\end{aligned}
$$

\section{MATTER FORMATION}

During the explosion of the Big Bang, occurred as a result of the appearance of the first quantum, i.e. an elementary particle of energy that disturbed the absolute balance/equilibrium of the zero ideal continuum, a large amount of energy was released and transformed. The spacetime component of the universe arose as a result of the appearance of matter, i.e. another by-product of the transformation of dark energy or anti-energy into energy and antimatter into matter (as the constituent elements of the ideal continuum), at the momentums when the velocity of a quantum was higher than the speed of light. This phenomenon can be roughly explained by the so-called sonic boom phenomenon in a medium such as air, whereby this incompressible flow becomes compressible at high speeds (Figure 4).

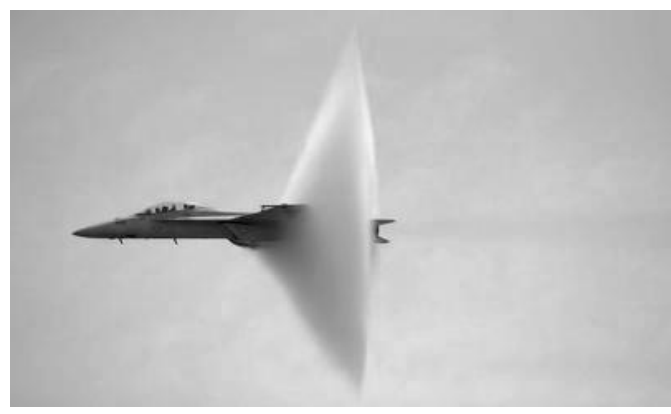

Fig 4:- Sonic Boom

\section{SPACE}

Space, in general, can also be defined as a product/derivative of the formation of matter from energy wide-spread in the vicinity of the ideal continuum (the space of the ideal continuum is considered to be completely condensed and non-reference space). In general, if existent, space is measurable and n-dimensional (although it is usually interpreted as three-dimensional). If it is measurable, it is then also bordered by gravitationalsheds. As already mentioned in the previous chapters, time and time measurement in such space is relativistic, and is closely related to its gravity, and completely irrelevant in other types of space and systems. The time component, contained in the origin of its n-dimensional coordinate system (Figure 5), forms a unique space-time frame, based on its gravity. In such a system, even without external influences such as gravitational waves and/or some other cosmic events (galaxy collapse, star explosion, etc.), according to the already known laws of quantum physics, space or the so-called environment of the ideal continuum can produce the ideal continuum (such as black holes). 


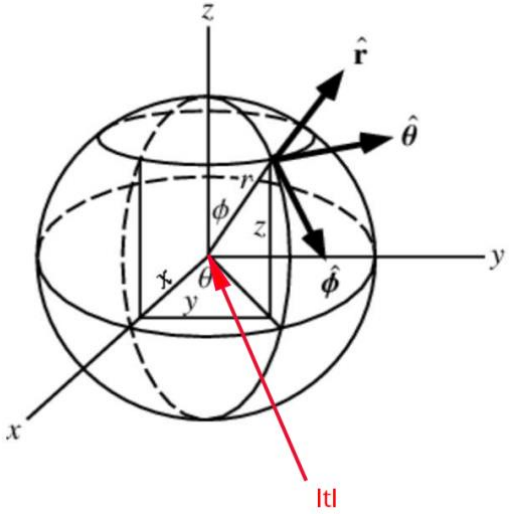

Fig 5:- Absolute n-dimensional (spherical) coordinate system with time coordinate / $\mathrm{t} /$ at its origin

\section{ENERGY BALANCE IN THE IDEAL CONTINUUM AND THE UNIVERSE}

Before the beginning of the universe, we can say that apart from the medium we have defined as the ideal continuum of infinitesimal or infinite dimensions, there was no visible energy, nor matter, nor gravity, nor space-time (6). The interior and the environment of the ideal continuum consisted of infinite amounts of dark energy (anti-energy) and antimatter $(|\Gamma|)$, which we will present and equalize with finite and integer number 1 (7).

$$
\begin{gathered}
|0|=|0| \\
|\Gamma|=1
\end{gathered}
$$

At the so-called zero momentum, when the absolute equilibrium or the equilibrium of the ideal continuum is disturbed by the appearance of the first quantum of energy or the photon of light (initial energy $|\Delta|$ ), which stimulates the creation of the visible universe, the balance equation of total energy starts taking shape (8).

$$
|\Gamma|+|\Delta|=1
$$

This event is also known by the so-called Big Bang. Immediately after that, once the energy velocities of antimatter particles exceed the speed of light, all other forms and derivatives of energy arise and get released at the atomic and subatomic level (of quantum energy $|\mathrm{A}|$ ), i.e. at a higher level, of matter or the so-called energy collectors (the energies of matter bodies $|\mathrm{B}|$ ) simultaneously creating space-time. As a result of energy arising from dark energy (anti-energy) and matter from anti- matter, the balance equation of energy gets two new components, i.e. (9).

$$
|\mathrm{A}|+|\mathrm{B}|+|\Gamma|+|\Delta|=1(9)
$$

As the reference system from which we observe all these events trying to establish their mutual laws, affects our understanding of the described chain of events, in accordance with the established distortion of the newly formed space-time, we must correct the balance equation of the total energy in the universe with physical constants (10).

$$
\begin{gathered}
|\mathrm{A}| f(\alpha, \beta, \gamma, \delta)+|\mathrm{B}| f(\beta, \alpha, \gamma, \delta)++|\Gamma| f(\gamma, \alpha, \beta, \delta)+ \\
|\Delta| f(\delta, \alpha, \beta, \gamma)=1 \quad(10)
\end{gathered}
$$

If we combine all physical constants and their arithmetic errors into one universal physical constant $(\eta)$ (11), or we combine all energy components except the initial (13), we get the following expressions for the total energy of the universe in a developed and general form (12, 14).

$$
\begin{gathered}
\eta=\Sigma f(\alpha, \beta, \gamma, \delta) \\
\eta(|\mathrm{A}|+|\mathrm{B}|+|\Gamma|+|\Delta|)=1 \\
\Sigma E=|\mathrm{A}|+|\mathrm{B}|+|\Gamma| \\
\eta(\Sigma E+|\Delta|)=1
\end{gathered}
$$

The following table presents some of the possible values of individual components of the total energy balance, as well as the theoretical assumptions of their approximate and relative relations over time, without any experimental confirmation or specific observations (Table 1).

\begin{tabular}{|c|c|c|c|c|c|}
\hline \multirow{2}{*}{ Momentum } & $\begin{array}{c}\text { Quantum } \\
\text { Energy }\end{array}$ & Matter Energy & $\begin{array}{c}\text { Anti-energy and } \\
\text { Antimatter } \\
\text { Energy }\end{array}$ & Initial Energy & \multirow{2}{*}{ Total } \\
\cline { 2 - 5 } & $|\mathrm{A}|$ & $|\mathrm{B}|$ & $|\Gamma|$ & $|\Delta|$ & \multirow{2}{*}{1} \\
\hline Absolute Equilibrium & 0 & 0 & 1 & 0 & \multirow{2}{*}{$\mathbf{1}$} \\
\hline $\begin{array}{c}\text { Zero Momentum, Initial } \\
\text { Energy Appearance }\end{array}$ & 0 & 0 & \multicolumn{2}{|c|}{$\mathbf{0 . 7 5}$} & 1 \\
\hline $\begin{array}{c}\text { Real Momentum, Today } \\
\text { (with the universal physical } \\
\text { constant } \eta \text { ) }\end{array}$ & \multicolumn{2}{|c|}{$\mathbf{0 . 2 5}$} & 1 & 0 & 1 \\
\hline Projection at the End & 0 & 0 & 1 & 0 \\
\hline
\end{tabular}

Table 1:- The components of the total energy balance and their approximate and relative relations 


\section{CONCLUSIONS}

From all the mentioned and analysed in the previous chapters, we can draw more several very important conclusions, namely:

that the total energy complies to the general law on energy sustainability, in all its forms and states;

that time is relative, and also a product of the appearance of matter and gravity under the influence of which the space-time distortion occurred;

$>$ that the physical constants, by which we are trying to calibrate our mathematical and physical models of the universe in relation to the reference system of the observer, are also the product of the space-time arose;

that energy is indestructible and that the universe is eternal, and that it existed even before the events of the so-called Big Bang and that it will exist even after its complete disintegration or inflation, i.e. the transformation of matter into antimatter and energy into anti-energy.

Eventually, we can also conclude that the physics of the ideal continuum, quantum physics and classical physics of the material body, actually form a single, unique physics, i.e. a universal science that studies energy states and phenomena in the entire, visible and invisible, universe. If we compared the specifics of these three scientific disciplines, we could highlight the following:

that the physics of the ideal continuum is the physics of events and information, and that quantum physics is the physics of all energy derivatives resulting from the transformation of anti-energy into energy (radiation, waves, light) and vice versa, i.e. classical physics is the physics of matter and matter bodies (energy collectors);

that the physics of the ideal continuum is the physics in which the basic physical quantities reach infinite values (4) or are equal to zero (5), and that quantum physics is the physics in which the basic physical quantities reach their finite and maximum values (15), i.e. classical physics is the physics in which the basic physical quantities never reach their final and maximum values (16) until they get in touch or come into a direct interaction with the ideal continuum.

$$
\begin{aligned}
& |v| \cong c \\
& |v| \ll c
\end{aligned}
$$

that the physics of the ideal continuum is the physics of medium, energy states and the processes without spacetime, and that quantum physics is the physics of energy states and processes at the atomic and subatomic level in greater space-time, on the basis of their presence the appearance of the ideal continuum is possible within that space-time, i.e. classical physics is the physics of matter and matter bodies in space-time.

\section{REFERENCES}

[1]. G. Lemaître (1946), The Primeval Atom - an Essay on Cosmogony, D. Van Nostrand Co. 